\title{
Hereditary thermosensitive neuropathy
}

INSERM

\section{Source}

INSERM. (1999). Orphanet: an online rare disease and orphan drug data base. Hereditary thermosensitive neuropathy. ORPHA:84093

Hereditary thermosensitive neuropathy is a rare, demyelinating, hereditary motor and sensory neuropathy characterized by reversible episodes of ascending muscle weakness, paresthesias and areflexia triggered by a febrile episode, with or without pressure palsy. 